\title{
Dexamethasone Infusion Testing in the Diagnosis of Cushing's Syndrome
}

\author{
HUY ANH TRAN AND NIKOLAI PETROVSKY*
}

Hunter Area Pathology Service, John Hunter Hospital, Locked Bag Number 1, Hunter Mail Region Centre, New South Wales, Australia 2310

*Autoimmunity Research Unit, Australian National University Medical School, The Canberra Hospital, Woden, Australian Capital Territory, Australia 2606

\begin{abstract}
Cushing's syndrome and its various aetiologies is a markedly difficult diagnosis to make given its subtle signs, sometime cyclical nature, and the lack of a single definitive diagnostic test. Although a great variety of diagnostic tests have been developed to assist in the diagnosis, even with the best clinical acumen, biochemistry and medical imaging the diagnosis can remain elusive. The long low and high dose oral dexamethasone suppression test is cumbersome, costly and often requiring an extended inpatient stay. The utility of the dexamethasone suppression test would be greatly enhanced if it could be performed as a short outpatient procedure. In this study we sought to confirm and refine the clinical utility of the high dose $4 \mathrm{mg}$ intravenous dexamethasone suppression test as an alternative diagnostic test for Cushing's syndrome. There were a total of 31 subjects: 8 patients with proven pituitary Cushing's disease, 3 with primary adrenal tumors, 10 with pseudo-Cushing's syndrome and 10 healthy controls. All subjects with pseudo-Cushing's syndrome suppress serum cortisol at +5 and at +24 hours. In subjects with pituitary Cushing's disease, 7 out of $8(88 \%)$ had serum cortisol suppressed at +5 hours but rebounded at +24 hours to at least $70 \%$ of the original serum level. Primary adrenal tumors showed a pattern of non-suppression throughout. The $4 \mathrm{mg}$ intravenous dexamethasone suppression test is excellent in ruling out pseudo-Cushing's syndrome. This test is much simpler and more convenient than the oral dexamethasone suppression test in confirming clinical suspicion of pituitary Cushing's disease.
\end{abstract}

Key words: Diagnostic tests, Intravenous, Dexamethasone, Cushing's syndrome

(Endocrine Journal 52: 103-109, 2005)

ENDOGENOUS Cushing's syndrome (CS) has several aetiologies including pituitary-dependent Cushing's disease (PC), ectopic ACTH syndrome (EAS), primary adrenal tumour (PAT) and cyclical Cushing's (CC). Assessment is complicated by the possibility of pseudoCushing's syndrome (Pseudo-CS) including the effects of depression on the hypothalamic-pituitary-adrenal axis. The low and high dose oral dexamethasone suppression tests (ODST) are important procedures in the diagnostic workup but these standard four-day oral dexamethasone tests are time consuming, costly and

Received: August 23, 2004

Accepted: November 19, 2004

Correspondence to: Dr. Huy Anh TRAN, Department of Clinical Chemistry, Hunter Area Pathology Service, John Hunter Hospital, New South Wales, Australia 2310 require an inpatient stay. Attempts to evaluate the utility of intravenous dexamethasone infusion in the diagnosis of CS scatter throughout the past few decades [1-3]. Over the years, there has been a number of evaluation of an alternative one-day outpatient procedure using intravenous dexamethasone infusion. The dosage of infusions include $1.75 \mathrm{mg}(5 \mu \mathrm{g} / \mathrm{kg} / \mathrm{hr})$ for 5 hours [4], $4 \mathrm{mg}(1 \mathrm{mg} / \mathrm{hr})[5,6], 7 \mathrm{mg}(1 \mathrm{mg} / \mathrm{hr})$ [7], $8 \mathrm{mg}$ bolus infusion [8] and $10.5 \mathrm{mg}(1.5 \mathrm{mg} / \mathrm{hr}$ for 7 hours) [9]. In this study we seek to confirm and refine the clinical utility of the dexamethasone infusion test as an alternative diagnostic test in the clarification of various causes of Cushing's syndrome. 


\section{Patients and Methods}

The test was performed in 31 consented subjects.

There were 10 normal subjects ( 6 females (F) and 4 males (M)). The mean age \pm standard error of the mean (SEM) was $44.9 \pm 4.4$ years, range 25 to 66 years. The mean body mass index was $23.2 \pm 2.6 \mathrm{~kg} / \mathrm{m}^{2}$. All normal female subjects report regular menses ranging from 24 to 34 days between cycles.

The following criteria (at least 4 out of 5) are to be met for the diagnosis of PC to be fulfilled:

- Clinical and biochemical evidence of endogenous hypercortisolaemia.

- Confirmation of pituitary adenoma by pituitary Magnetic Resonance Imaging (MRI).

- Evidence of pituitary-dependent ACTH overproduction using bilateral simultaneous inferior petrosal sinus sampling (BIPPS).

- Resolution of hypercortisolaemia after surgery requiring glucocorticoid therapy.

- (Immuno-) histological confirmation of the presence of ACTH in pituitary tissue (available in some cases only).

There were 10 ( $7 \mathrm{~F}: 4 \mathrm{M})$ subjects with PC satisfying the aforementioned criteria. Five female patients had oligomenorrhoea with periods ranging from 40 to 88 days between cycles. The other two patients were receiving the oral contraceptive pills.

Three patients $(2 \mathrm{~F}: 1 \mathrm{M})$ with PAT were studied. All had surgically and histologically proven adrenal adenoma. The removed glands were 35,45 and 42 grams in weight respectively. Both females in this group reported amenorrhoea in the preceding 4 months.

Ten patients ( $8 \mathrm{~F}: 2 \mathrm{M}$ ) with pseudo-CS were referred for further investigations following the finding of one or a combination of elevated morning serum cortisol, elevated 24 hour urinary free cortisol or loss of diurnal cortisol pattern. Six (F) had depressive illness, 2 (F) had elevated urinary cortisol excretion, 1 (M) suffered from alcoholism and 1 (M) had lost the diurnal cortisol rhythm. Six female patients had oligomenorrhoea with periods ranging from 38 to 98 days between cycles. Pseudo-CS was confirmed and PC, including CC, was excluded (at 4 out of 5) in all these subjects based on the following criteria:

- Low clinical suspicion of Cushing's syndrome.

- Prolonged duration of follow-ups: at 2 years at the time of this report with no clinical suspicion of hypercortisolaemia.
- The presence of co-morbidities including obesity, depression and anxiety disorders.

- Normal response to a $1 \mathrm{mg}$ overnight ODST.

- Normal pituitary MRI in some cases.

\section{Intravenous (IV) dexamethasone infusion protocol}

Two intravenous (IV) cannulas were inserted for IV infusion of dexamethasone and venesection. Starting at 11:00 hours on the day of procedure, dexamethasone $4 \mathrm{mg}$ was given by IV infusion at $1 \mathrm{mg} / \mathrm{hr}$ according to the Abou Samra protocol [5]. Samples were taken for serum cortisol at T- $1,0,+3,+4,+5,+22$ and +24 hours. In order to refine and circumvent the need for inpatient hospitalisation, no sampling was performed in the 17 hour period between +5 and +22 hours. Previous report [8] was reassuring in that levels at +22 and +24 hours reflected accurately that which occurred in the unmonitored time gap in the various causes of CS.

\section{Serum cortisol assay}

Serum cortisol and ACTH assays were measured using the Immulite 2000 platform (Diagnostic Products Services (DPC), Los Angeles, California, USA) with the co-efficient of variations of $5.5 \%$ and $8.6 \%$ respectively. The detection limit for serum cortisol was $<28 \mathrm{nmol} / \mathrm{L}$.

\section{Statistical analyses}

Results are presented as the Mean \pm Standard Error (SE) and Confidence Intervals (CI).

Statistical analyses including $\mathrm{CI}$ are performed with the software package Confidence Interval Analysis (Version 2.0.0) provided with reference 10.

\section{Results}

The normal control subjects $(n=10)$ showed an expected response to intravenous dexamethasone with continuous suppression of serum cortisol at $+5,+22$ and +24 hours. The 24 hour values were all suppressed to below detection capability of the assay.

In the subjects with pseudo-CS $(n=10)$, the range of hypercortisolaemia was broad. Similar to normal subjects however, suppression occurred at +5 hours, +22 
Table 1. Shows individual cortisol concentrations at the critical time points in the various categories of CS including the Mean, SEM, 95\% CI and percentage of suppression at $+5,+22$ and +24 hours (compared with baseline) respectively.

\begin{tabular}{|c|c|c|c|c|c|}
\hline & \multicolumn{5}{|c|}{ TIME (HRS) } \\
\hline & -1 & 0 & +5 & +22 & +24 \\
\hline & \multicolumn{5}{|c|}{ A. Normal Subjects $(\mathrm{NS})-(\mathrm{n}=10)$} \\
\hline & \multicolumn{5}{|c|}{ Serum Cortisol concentration $(\mathrm{nmol} / \mathrm{L})$} \\
\hline 1 & 421 & 116 & 41 & 31 & 28 \\
\hline 2 & 368 & 129 & 82 & 30 & 32 \\
\hline 3 & 228 & 83 & 55 & 28 & 28 \\
\hline 4 & 556 & 121 & 72 & 42 & 28 \\
\hline 5 & 481 & 101 & 66 & 30 & 28 \\
\hline 6 & 391 & 92 & 51 & 28 & 28 \\
\hline 7 & 310 & 88 & 48 & 33 & 32 \\
\hline 8 & 288 & 92 & 57 & 34 & 28 \\
\hline 9 & 411 & 79 & 60 & 28 & 28 \\
\hline 10 & 251 & 81 & 62 & 28 & 28 \\
\hline Mean & 370.5 & 98.2 & 59.4 & 31.2 & 28.8 \\
\hline$S E M$ & 32.7 & 5.6 & 3.8 & 1.4 & 0.53 \\
\hline \multicolumn{3}{|c|}{ Percentage of suppression } & $84 \%$ & $92 \%$ & $92 \%$ \\
\hline \multirow[t]{2}{*}{$95 \% C I$} & $\begin{array}{l}296.5 \text { to } \\
444.5\end{array}$ & $\begin{array}{l}85.4 \text { to } \\
111.0\end{array}$ & $\begin{array}{l}50.9 \text { to } \\
68.0\end{array}$ & $\begin{array}{l}28.1 \text { to } \\
34.3\end{array}$ & $\begin{array}{l}27.6 \text { to } \\
30.0\end{array}$ \\
\hline & \multicolumn{5}{|c|}{ B. Pseudo-Cushing's syndrome (Pseudo-CS) $-(\mathrm{n}=10)$} \\
\hline $1^{*}$ & 926 & 652 & 206 & 107 & 180 \\
\hline 2 & 351 & 151 & 76 & 28 & 28 \\
\hline $3 *$ & 1136 & 1007 & 380 & 112 & 140 \\
\hline 4 & 580 & 440 & 250 & 210 & 190 \\
\hline 5 & 190 & 160 & 40 & 28 & 28 \\
\hline 6 & 403 & 90 & 30 & 28 & 28 \\
\hline 7 & 195 & 180 & 52 & 28 & 28 \\
\hline 8 & 594 & 313 & 98 & 68 & 28 \\
\hline 9 & 270 & 159 & 70 & 30 & 28 \\
\hline 10 & 708 & 270 & 110 & 101 & 88 \\
\hline Mean & 535.3 & 342.2 & 131.2 & 74 & 76.6 \\
\hline$S E M$ & 100.3 & 91.1 & 35.7 & 18.9 & 21.6 \\
\hline \multicolumn{3}{|c|}{ Percentage of suppression } & $75 \%$ & $86 \%$ & $86 \%$ \\
\hline \multirow[t]{3}{*}{$95 \% C I$} & $\begin{array}{l}308.4 \text { to } \\
762.2\end{array}$ & $\begin{array}{l}136.1 \text { to } \\
548.3\end{array}$ & $\begin{array}{l}50.4 \text { to } \\
212.0\end{array}$ & $\begin{array}{l}31.2 \text { to } \\
116.8\end{array}$ & $\begin{array}{l}27.8 \text { to } \\
125.4\end{array}$ \\
\hline & \multicolumn{5}{|c|}{ C. Pituitary Cushing's disease $(\mathrm{PC})-(\mathrm{n}=8)$} \\
\hline & \multicolumn{5}{|c|}{ Serum cortisol concentration $(\mathrm{nmol} / \mathrm{L})$} \\
\hline 1 & 412 & 612 & 141 & 381 & 350 \\
\hline 2 & 350 & 378 & 98 & 550 & 490 \\
\hline 3 & 700 & 530 & 80 & 550 & 490 \\
\hline 4 & 510 & 410 & 112 & 432 & 411 \\
\hline $5^{*}$ & 870 & 811 & 750 & 898 & 911 \\
\hline 6 & 1211 & 702 & 128 & 718 & 650 \\
\hline 7 & 740 & 712 & 200 & 376 & 381 \\
\hline 8 & 665 & 562 & 68 & 401 & 528 \\
\hline Mean & 682.3 & 589.6 & 197.1 & 538.3 & 526.4 \\
\hline$S E M$ & 97.5 & 53.2 & 80.3 & 65.9 & 64.3 \\
\hline \multicolumn{3}{|c|}{ Percentage of suppression } & $71 \%$ & $21 \%$ & $22 \%$ \\
\hline \multirow[t]{2}{*}{$95 \% C I$} & $\begin{array}{l}451.8 \text { to } \\
912.7\end{array}$ & $\begin{array}{l}463.8 \text { to } \\
715.4\end{array}$ & $\begin{array}{l}7.3 \text { to } \\
387.0\end{array}$ & $\begin{array}{l}382.4 \text { to } \\
694.1\end{array}$ & $\begin{array}{l}374.4 \text { to } \\
678.4\end{array}$ \\
\hline & \multicolumn{5}{|c|}{ D. Primary Adrenal Tumors $(\mathrm{PAT})-(\mathrm{n}=3)$} \\
\hline 1 & 301 & 270 & 510 & 590 & 600 \\
\hline 2 & 1460 & 1370 & 1201 & 2011 & 1600 \\
\hline 3 & 660 & 780 & 880 & 900 & 820 \\
\hline Mean & 807 & 806.7 & 863.7 & 1167 & 1006.7 \\
\hline SEM & 342.6 & 317.8 & 199.6 & 431.4 & 303.4 \\
\hline
\end{tabular}

and +24 hours. Subjects number 1 and 3 (denoted $*$ in section $\mathrm{B}$, Table 1) were unusual with slight increases in cortisol levels at +24 hours compared with +22 hours rather than continued suppression. These patients however fully satisfied the aforementioned criteria for the diagnosis of pseudo-CS. Given this result, this test thus had a specificity of $80 \%$ in the assessment of Pseudo-CS.

In subjects with confirmed PC $(\mathrm{n}=8)$, there was also suppression at +5 hours with rebound at +22 and +24 hours. The lowest value for serum cortisol at +22 hours was $376 \mathrm{nmol} / \mathrm{L}$ which did not overlapped with the values in the pseudo-CS group. Subject number 5 (denoted * in section $\mathrm{C}$, Table 1 ) showed an anomalous response with no suppression at +5 hours. As this subject was satisfied the diagnostic criteria for PC, the sensitivity of this test was $90 \%$.

In subjects with adrenal adenoma $(n=3)$, there was a wide range of hypercortisolaemia. No suppression of cortisol was seen at all throughout the dynamic test. There was a suggestion of a cortisol rise at +24 hours but the number was small. Serum ACTH was suppressed below the detection limit throughout the test.

All individual data at the critical time points are listed in Table 1. The broad CI's are not shown for the PAT group as the patient number is small for this analysis. Fig. 1 summarises the mean cortisol response for all categories.

\section{Discussion}

Endogenous CS presents as one of the most difficult diagnostic problems in clinical endocrinology. There is no single gold standard test and the diagnosis relies on a combination of clinical manifestation, biochemical testings and response to therapy, be it pituitary surgery or otherwise. There are vast numbers of tests used to elucidate the cause of CS after hypercortisolaemia has been confirmed biochemically [12-14]. The fact that there are numerous tests for this condition reinforces the belief that no one test is diagnostic.

These tests can be arranged into first-line screening tests which confirm or exclude the presence of hypercortisolaemia in CS. These include the 24-hour urinary free cortisol level, timed overnight cortisol/Creatinine ratio [15], the $1 \mathrm{mg}$ oral overnight dexamethasone suppression test and the single midnight serum cortisol level [13], all of which have sensitivities and specifici- 


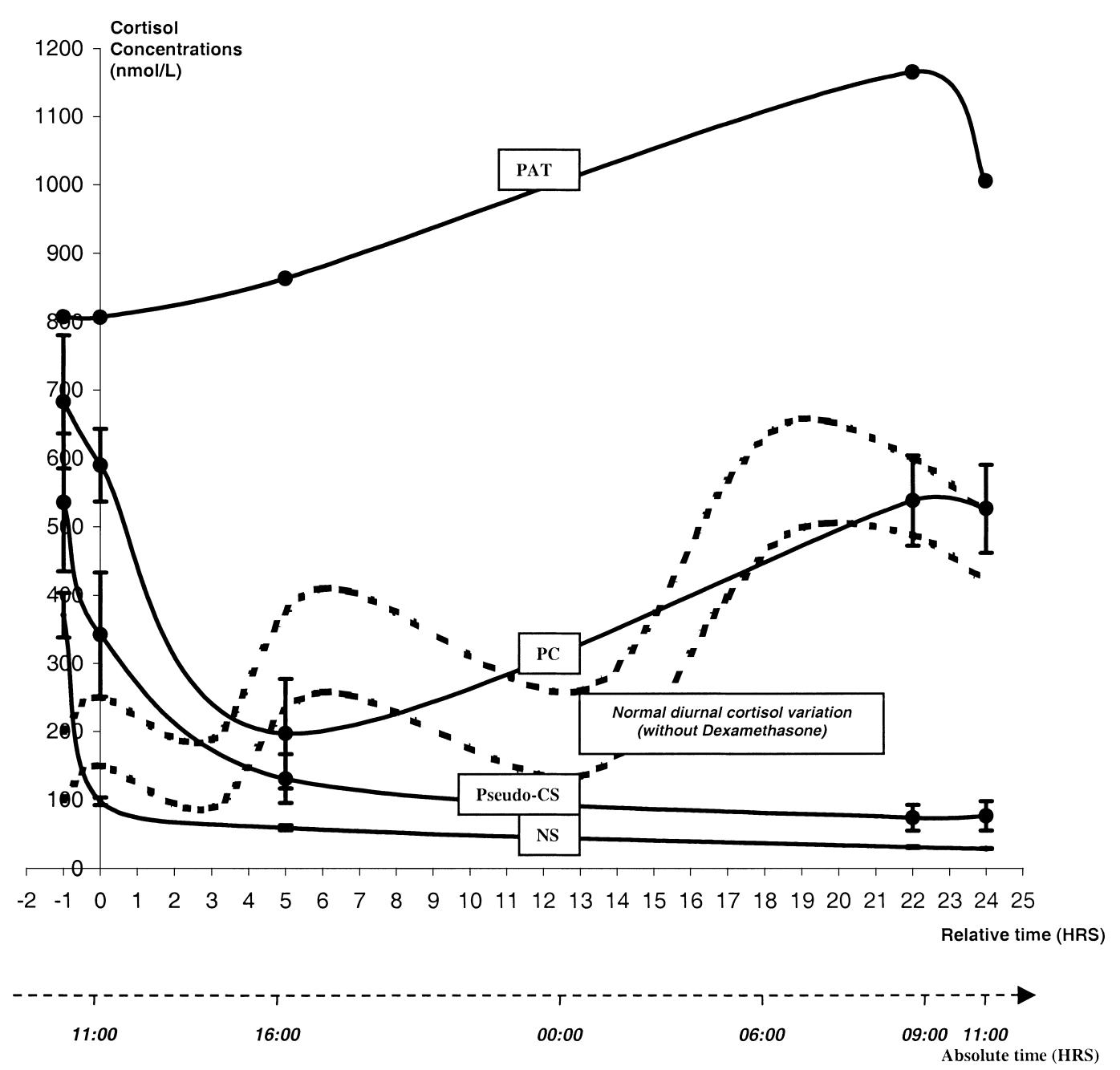

Fig. 1. Illustrates the different responses to IV Dexamethasone infusion in the various categories of Cushing's syndrome using mean values at critical time points. Error bars indicate SEM. Broken lines indicate the cortisol diurnal variation pattern in normal subjects without Dexamethasone (adapted from reference 11).

ties ranging between $95-100 \%$ and $94-98 \%$, respectively. Newer first-line tests include the combined oral low dose Dexamethasone suppression test with IV Corticotropin Releasing Hormone (CRH) and IV Desmopressin stimulation test which help to differentiate between Pseudo-CS and CS [12, 16, 17]. These of course are complex and require hospital admission to ensure the correct procedures are followed.

The second-line tests are more complex and are used for the diagnostic work up of the various causes of CS. Some of these include the single ACTH assay, the oral high dose dexamethasone suppression test (HDDST), CRH stimulation test with or without bilateral inferior petrosal sinus sampling (BIPSS) [12, 14]. These dynamic biochemical tests, of course, should be compli- mented with the appropriate diagnostic imaging, most usually by MRI [13].

The most commonly used second-line test is the high dose oral dexamethasone suppression test (HDDST) where $2 \mathrm{mg}$ of oral dexamethasone is given every 6 hours for 48 hours starting at 09:00 hours with serum and urinary cortisol levels taken at regular intervals. This test has a sensitivity and specificity of $81 \%$ and $65 \%$ for the diagnosis of PC respectively [18]. This test entails a complex regimen that requires the cooperation of both patient and nursing staff. Regimen confusion, patient compliance and variability in dexamethasone metabolism can confound the result [19]. Absorption and drug metabolism abnormalities are highly pertinent to tests that involve the use of oral 


\section{SUGGESTED ALGORITHM USING IV DEXAMETHSONE INFUSION TESTING}

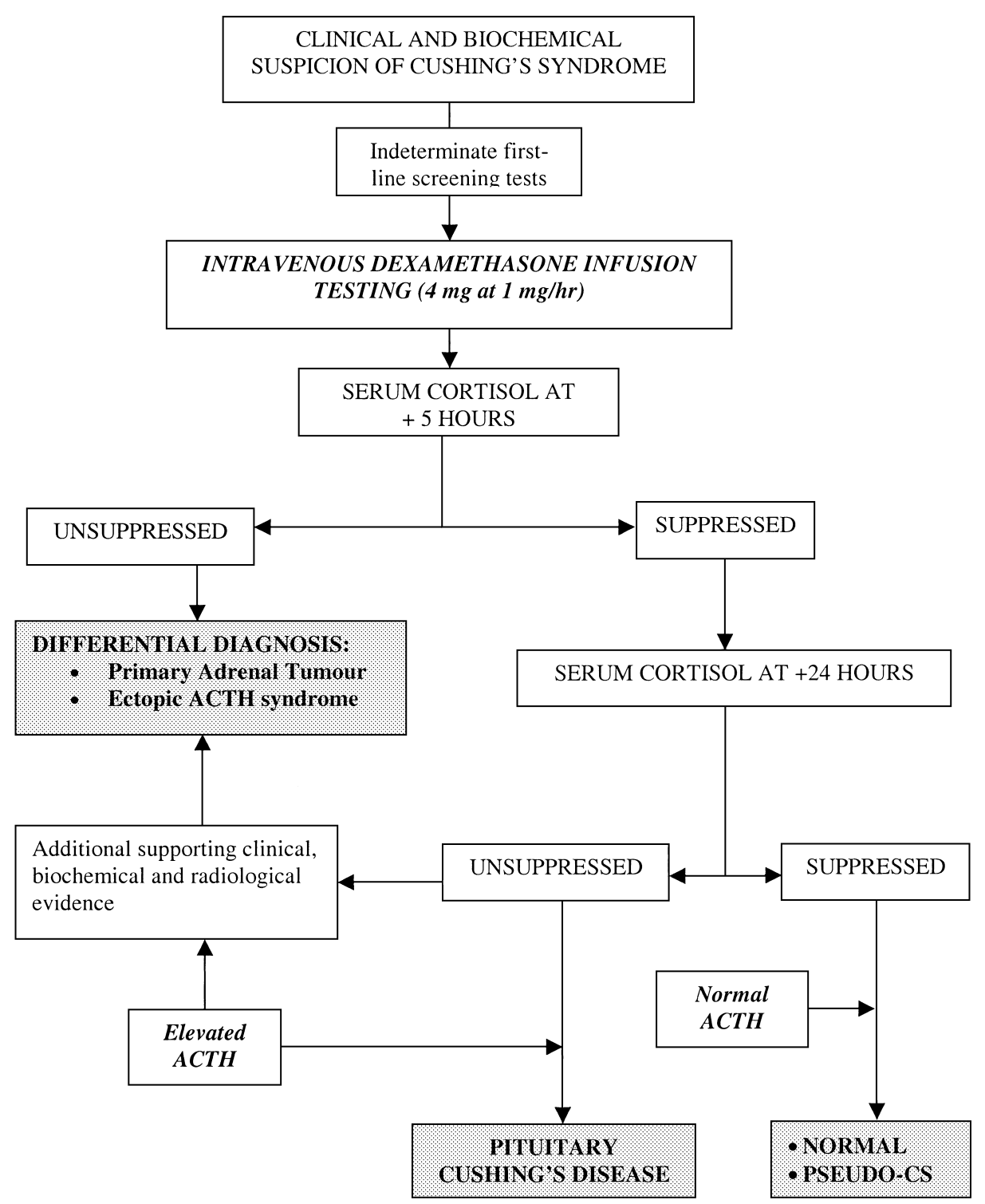

Fig. 2. Algorithm allocating the place of the Dexamethasone infusion test in the diagnostic work-up for CS. This test should be complimented by clinical, additional biochemical and radiological evidence.

medications. In the cases of LDDST and HDDST, both poor absorption and fast metabolisers, either inherited or induced, can lead to false non-suppression and subsequent misdiagnosis [20]. In addition, these tests are not recommended in acute illness due to heightened cortisol response [21].

In order to circumvent the confounders and complexity of oral testing, many have adopted the dexamethasone infusion test but with variable doses ranging from $1.75 \mathrm{mg}$ to $10.5 \mathrm{mg}$ (with $4 \mathrm{mg}$ being most commonly used) [4-7, 9] and $8 \mathrm{mg}$ IV bolus injection [8]. The common pattern to all these unstandardised studies was suppression in normal subjects and PC at between +5 to 7 hours. PC cortisol levels rebounded invariably to baseline level at +24 hours. In this study we have added evidence to show that a $4 \mathrm{mg}$ dexamethasone infusion test has diagnostic utility in subjects referred for the clarification of CS. These results concur with and 
support previous findings in showing that the dexamethasone infusion test is useful in the differential diagnosis of CS.

In our study, the absolute level of cortisol suppression at +5 and +24 hours in normal subjects was $<30 \mathrm{nmol} / \mathrm{L},<83 \mathrm{nmol} / \mathrm{L}$ in the Abou Samra series [5], and $<51 \mathrm{nmol} / \mathrm{L}$ in the LeClerc et al. series [6]. In abnormal subjects who were deemed to have pseudo-CS, the expected response would be an $80 \%$ decrease from baseline cortisol level at +5 and +24 hours. In addition, there was a statistically (though not clinically) significant difference of serum cortisol at +24 hours between NS and Pseudo-CS using 95\% CI. One possibility was the presence of $\mathrm{CC}$, a particularly difficult diagnosis to make as cycling cortisol levels could range between 12 hours to 85 days [22]. The dimension of time, regular follow-ups (2 years at the time of this analysis) and detailed correlation between clinical and biochemical parameters confidently delivered the extreme reassurance that $\mathrm{CC}$ was a remote possibility in this cohort. Another and more plausible alternative was the higher cortisol set point in pseudo-CS and relative resistance to dexamethasone [14]. This explained the difference in mean cortisol concentrations between the two groups which, despite physiological difference, retained the common pattern of response.

All patients with PC had their serum cortisol suppressed, at least partially, at +5 hours following intravenous dexamethasone. The rebound of serum cortisol the following day at +22 and +24 hours was a consistent and reliable diagnostic marker. Abou Samra [5] suggested $50 \%$ reduction from baseline value at +5 hours and rebound to $>276 \mathrm{nmol} / \mathrm{L}$ at +24 hours. Van den Bogaert [7] adopted a cortisol level of more than $190 \mathrm{nmol} / \mathrm{L}$ at +7 hours to identify patients with PC. In our series, the majority of patients with PC were confirmed by histopathology, the rest by clinical and biochemical response to surgery. The observed response was more than $50 \%$ and less than $20 \%$ reduction from baseline cortisol value at +5 and +24 hours respectively. This would present a specificity of $90 \%$.

In PAT, serum cortisol failed to suppress at any time point in response to dexamethasone infusion test and this was consistent with previous reports [5-9]. All three patients had typical clinical phenotype of cortisol excess confirmed by high urinary cortisol excretion and undetectable ACTH. There was no adrenal carcinoma but the pattern of response was expected to be identical with non-suppression. The diagnosis of PAT would normally be straightforward based on the finding of extremely high serum cortisol but with variable set points, as shown in this study, with undetectable ACTH levels supported by positive radiological imaging. Thus the value of this dynamic test was limited, did not add to the diagnosis and probably remained of academic interest.

EAS is relatively rare and reflecting its absence in this original study. The total number of EAS cases undergoing the dexamethasone infusion test as reported in the literature in the last 24 years amounts to 18 with timed samples at either +5 to 7 hours and/or +24 hours [5-9]. The predominant response noted was nonsuppression. Henceforth and based on reported literature, it is our suggestion that this test be used primarily in distinguishing PC from EAS. In this situation, a single sample at +5 hours is pivotal in separating the two conditions. The cortisol concentration here is expected to be greater than $50 \%$ reduction from baseline for PC compared with non-suppression in EAS. The +24 hour sample is unhelpful in this particular situation as both will be elevated. Normal subjects will, presumably, not be needing to proceed to this dynamic test unless first line screening tests are equivocal. The diagnosis of PAT is mostly straightforward as previously mentioned.

A possible algorithm is suggested in Fig. 2 for the differential diagnosis of CS. Although mentioned briefly, ACTH level remained crucial in the distinguishing between adrenal from non-adrenal causes of CS. Dexamethasone infusion testing can then be used to identify if PC is the source of hypercortisolaemia.

\section{Summary}

The $4 \mathrm{mg}$ intravenous dexamethasone infusion test is consequently a useful diagnostic test for PC. It is useful in providing differential diagnostic information in patients with CS. It is simpler, more convenient and can be used without loss of diagnostic utility and with less consumption of valuable resources. 


\section{References}

1. James VH, Landon J, Wynn V (1965) Oral and intravenous suppression tests in the diagnosis of Cushing's syndrome. J Endocrinol 33: 515-524.

2. Croughs RJ, Docter R, de Jong RH (1973) Comparison of oral and intravenous dexamethasone suppression tests in the differential diagnosis of Cushing's syndrome. Acta Endocrinol (Copenh) 72: 54-62.

3. Linquette M, Cappoen JP, Fourlinnie JC, Harpignies G (1980) Test of pituitary adrenal restraining with IV dexamethasone in the diagnosis of Cushing's syndrome. Sem Hop 56: 1380-1384 (Abstract only).

4. Atkinson AB, Mcateer EJ, Haden DR, Kennedy L, et al. (1989) A weight-related intravenous dexamethasone suppression test distinguishes obese controls from patients with Cushing's syndrome. Acta Endocrinol (Copenh) 120: 753-759.

5. Abou Samra AB, Dechaud H, Estour B, Chalendar D, et al. (1985) Beta-Lipotropin and Cortisol responses to an Intravenous infusion Dexamethasone Suppression test in Cushing's Syndrome and Obesity. J Clin Endocrinol Metab 61: 116-119.

6. Leclerc I, Bolte E, Lacroix A (1997) Intravenous Dexamethasone Suppression test for the differential diagnosis of Cushing's syndrome. Abstract from the Proceedings of the American Endocrine Society 1997.

7. Van den Bogaert DPM, de Herder WW, de Jong FH, Biemond P, et al. (1999) The continuous 7-hour intravenous dexamethasone suppression test in the differential diagnosis of ACTH-dependent Cushing's syndrome. Clin Endocrinol 51: 193-198.

8. Shilo S, Rosler A (1995) Single intravenous bolus of Dexamethasone for the differential diagnosis of Cushing's syndrome. J Pediatr Endocrinol Metab 8: 27-33.

9. Gambardella S, Tamburrano G, Giaccari A, Frontoni S, et al. (1989) Intravenous dexamethasone and subsequent $\mathrm{ACTH}$ test in comparison with dexamethasone oral test in the diagnosis of Cushing's syndrome: a report of 20 cases. J Endocrinol Invest 12: 163-170.

10. Newcombe RG, Altman DG (2000) Chapter 6, Proportions and their differences. In: Altman DG, Machin D, Bryant NT, Gardner MJ (ed.) Statistics with Confidence, $2^{\text {nd }}$ Edition. Br Med J Books 45-56.

11. Tran AH, Petrovsky N (2004) The peril of untimed serum cortisol measurement. Pathology 36: 279-281.

12. Arnaldi G, Angeli A, Atkinson AB, Bertagna X, et al. (2003) Diagnosis and Complications of Cushing's syndrome: A Consensus Statement. J Clin Endocrinol Metab 88: 5593-5602.

13. Orth D (1995) Cushing's Syndrome. N Engl J Med 332: 791-803.

14. Newell-Price J, Trainer P, Besser M, Grossman A (1998) The Diagnosis and Differential Diagnosis of Cushing's Syndrome and Pseudo-Cushing's States. Endocr Rev 19: 647-672.

15. Corcuff JB, Tabarin A, Rashedi M, Duclos M, et al. (1998) Overnight urinary free cortisol determination: A screening test for the diagnosis of Cushing's syndrome. Clin Endocrinol 48: 503-508.

16. Meier CA, Biller BM (1997) Clinical and biochemical evaluation of Cushing's syndrome. Endocrinol Metab Clin North America 26: 741-762.

17. Malerbi DA, Mendonca BB, Liberman B, Toledo SP, et al. (1993) The desmopressin stimulation test in the differential diagnosis of Cushing's syndrome. Clin Endocrinol (Oxford) 38: 463-472.

18. Aron DC, Raff H, Findling JW (1997) Effectiveness Versus Efficacy: The Limited Value in Clinical Practice of High Dose Dexamethasone Suppression Testing in the Differential Diagnosis of AdrenocorticotropinDependent Cushing's Syndrome. J Clin Endocrinol Metab 82: 1780-1785.

19. Leonard PK, Stewart MH, Meikle AW (1984) Cushing's Disease with 'Normal Suppression' due to Decreased Dexamethasone clearance. Arch Internal Med 144: 636-637.

20. Dimaraki VE, Jaffe AC (2003) Troglitazone induces CYP3a4 activity leading to falsely abnormal Dexamethasone suppression test. J Clin Endocrinol Metab 88: 3113-3116.

21. Sam S, Corbridge TC, Mokhlesi B, Comellas AP, Molitch ME (2004) Cortisol levels and mortality in severe sepsis. Clinl Endocrinol 60: 29-35.

22. Atkinson AB, McCance DR, Kennedy L, Sheridan B (1992) Cyclical Cushing's syndrome first diagnosed after pituitary surgery: a trap for the unwary. Clin Endocrinol 36: 297-300. 